\title{
Gender-specific nomogram models to predict the prognosis of male and female lung adenocarcinoma patients: a population-based analysis
}

\author{
Hui Wen ${ }^{1 *}$, Xuefeng Lin ${ }^{2 *}$, Daqiang Sun ${ }^{1,3}$ \\ ${ }^{1}$ Graduate School, Tianjin Medical University, Tianjin, China; ${ }^{2}$ Tianjin Medical College, Tianjin, China; ${ }^{3}$ Department of Thoracic Surgery, Tianjin \\ Chest Hospital of Nankai University, Tianjin, China \\ Contributions: (I) Conception and design: D Sun; (II) Administrative support: D Sun; (III) Provision of study materials or patients: X Lin; (IV) \\ Collection and assembly of data: X Lin, H Wen; (V) Data analysis and interpretation: H Wen, X Lin; (VI) Manuscript writing: All authors; (VII) \\ Final approval of manuscript: All authors. \\ \#These authors contributed equally to this work. \\ Correspondence to: Daqiang Sun. Department of Thoracic Surgery, Tianjin Chest Hospital of Nankai University, No. 261, Taierzhuang South Road, \\ Jinnan District, Tianjin 300000, China. Email: sdqmd@163.com.
}

Background: Epidemiological and clinical prognosis differences between male and female lung adenocarcinoma (LUAD) patients have been frequently reported. To improve prognosis determinations, gender-specific nomogram models should be developed and validated to predict the prognosis of patients with LUAD.

Methods: Using the Surveillance, Epidemiology, and End Results (SEER) database, LUAD patients diagnosed between 2010 and 2015 were used as SEER training and internal validation testing sets. Patients in Tianjin Chest Hospital with postoperative pathological diagnosis of LUAD from January 1, 2015 to October 1, 2016 were considered as Chinese external testing sets. Using the Kaplan-Meier method and log-rank tests, we compared all the included male and female LUAD patients' overall survival (OS) and lung cancerspecific survival (LCSS) rates. The female and male patients from SEER database were randomly divided into training and internal validation groups at a 7:3 ratio. Variables $(\mathrm{P}<0.05)$ in the multivariable LCSS Cox regression analysis were independent prognostic predictors of the nomogram models. Harrell's concordance index (C-index), calibration curves, decision curve analysis (DCA) curves, receiver operating characteristic (ROC) curves, and the area under the curves (AUCs) were used to test the calibration and accuracy of the gender-specific nomogram models.

Results: A total of 32,654 LUAD patients (17,372 females and 15,282 males) were identified. Ten variables [age, marital status, tumor site, differentiation grade, derived American Joint Committee on Cancer (AJCC) stage, tumor size, historic stage, surgery, derived AJCC N stage and chemotherapy] were statistically significant in the multivariate LCSS Cox regression analysis, and visualized through the nomogram models. The female and male training nomogram C-indexes were 0.827 and 0.811 , respectively. The 3- and 5-year AUCs of the LCSS were 0.881 and 0.872 in the female training set, respectively, and 0.879 and 0.881 in the male training set, respectively. The DCA results indicated that these nomogram models were excellent predictors of LUAD prognosis and can be used to supplement the prognostication of tumor, node, and metastasis (TNM) stage.

Conclusions: Given the different incidence and prognosis of LUAD between men and women, we developed gender-specific nomogram models with good discrimination and calibration capacity to predict 3and 5-year LUAD-specific survival.

Keywords: Nomogram model; prognosis; lung adenocarcinoma (LUAD); gender 
Submitted Aug 19, 2021. Accepted for publication Nov 17, 2021.

doi: $10.21037 / \mathrm{atm}-21-5367$

View this article at: https://dx.doi.org/10.21037/atm-21-5367

\section{Introduction}

Lung cancer is the leading cause of cancer-related death around the world $(1,2)$. In 2020, approximately 1.8 million people died from lung cancer worldwide. It is estimated that there will be 235,760 new lung and bronchus cancer cases in the United States in 2021 (3). To address this major global health problem, etiological treatment and prognosis are essential. In relation to smoking factors, epidemiological studies showed that in America, among patients with lung cancer, $19 \%$ were female non-smokers, $9 \%$ were male nonsmokers (4). In Japan and South Korea, about one-third of non-small lung cancer patients are non-smokers $(5,6)$. In addition to smoking, Jin et al. (7-9) believes that hormonal factors may play a role in the occurrence and development of lung cancer, and also in lung adenocarcinoma (LUAD) $(10,11)$. LUAD is one of the most prevalent pathological subtypes of lung cancer, and accounts for more than about one-third of lung cancers (12-14). Among lung cancer nonsmokers, the incidence of LUAD is higher in females than males (15-17), and gender is considered as an independent prognostic factor for $\operatorname{LUAD}(12,18)$. In a Swedish national cohort study, female LUAD patients generally had a better prognosis in lung cancer-specific survival (LCSS) across tumor, node, and metastasis (TNM) stages (19). Behrens et al. pointed women with LUAD have a higher density of tumor-associated immune infiltrating cells in their tumor tissues than male LUAD patients (20). Meanwhile, Woolston et al. believe female and male LUAD patients have different KRAS/EGFR mutations patterns, and effects of relevant copy number variation (cnv) may contribute to gender-specific prognosis of LUAD (21). Huang et al. (11) believe that estrogen promotes LUAD development through estrogen receptor beta (ER- $\beta$ ) pathway. Chen et al. (22) suggest that the ER- $\beta$ may play a significant role in LUAD progression via the mitogen-activated protein kinase kinase/ extracellular signal-regulated kinase signaling pathway; however, ER- $\beta$ overexpression in males indicates better survival (23). Wheatley-Price et al. (24) noted that female LUAD patients receiving platinum-based chemotherapy had more positive prognostic effects than male LUAD patients. Given the different LUAD incidence rates and prognosis between males and females, the prognosis of
LUAD for men and women need to be analyzed separately $(18,25)$. Nomograms, which are graphical models that predict the prognosis of cancer patients, have been shown to improve prognosis determinations and treatment stratifications in patients with thyroid carcinoma (26), uterine cervical carcinoma (27), colon cancer (28), and gastric carcinoma (29). Survival prediction nomogram tools demonstrate excellent predictive power in different cancers and medicine (30). However, to date, no gender-specific nomogram models for predicting the prognosis of male and female LUAD patients appear to have been established. Accordingly, there is a need for separately modeling the gender-specific prognosis of LUAD. For the purpose of achieving accurate individualized treatment, more clinical variables besides age and American Joint Committee on Cancer (AJCC) TNM stage should be incorporated to reach the individualized and efficient prediction. In this analysis, we gathered useful information about LUAD patients from the Surveillance, Epidemiology, and End Results (SEER) database. To supplement internal validation testing sets, pathologically diagnosed LUAD patients after operation in Tianjin Chest Hospital from January 1, 2015 to October 1, 2016 were taken into consideration. We then conducted a Cox regression analysis to identify relevant prognosticrelated factors and establish gender-specific nomogram models for male and female LUAD patients. These reliable and practical nomogram prediction models will be useful in providing individual treatment advice and improving clinical decisions.

We present the following article in accordance with the TRIPOD reporting checklist (available at https://dx.doi. org/10.21037/atm-21-5367).

\section{Methods}

\section{Ethical review}

This retrospective cohort study used data from the previously collected identifiable SEER database (the SEER-program, https://seer.cancer.gov) and Tianjin Chest hospital. All of the procedures performed in this study were conducted in accordance with the Declaration of Helsinki (as revised in 2013), and written informed consents have 
been provided by all included patients from Tianjin Chest Hospital. The study was approved by institutional ethics board of Tianjin Chest Hospital (ethical approval number: 2021LW-016).

\section{Study population}

In this retrospective study, data from SEER database were obtained using SEER*STAT 8.3.9 software according to the site code classifications (C34.0-34.9) and International Classification of Diseases for Oncology terrain code "8140/3: Adenocarcinoma NOS". Patients diagnosed with LUAD in SEER database from 2010-2015 were identified. Postoperative patients who were pathologically diagnosed with LUAD from January 1, 2015 to October 1, 2016 in Tianjin Chest Hospital were collected. To be eligible for inclusion in this study, participants had to meet the following criteria: (I) have complete medical information, including information about age, surgery methods, derived AJCC stage, derived AJCC N stage, tumor site, laterality, tumor historic stage, tumor size, and tumor differentiated grade; (II) have a pathologically confirmed diagnosis of LUAD; (III) have been diagnosed with primary malignant tumor (LUAD); and (IV) have accurate follow-up data. Patients were excluded from the study if information about their sex, age, laterality, tumor site, tumor differentiation grade, derived AJCC stage, derived AJCC N stage, tumor size, historic stage, and surgery was uncertain. A total of 32,654 patients (17,372 female LUAD patients and 15,282 male LUAD patients) met the inclusion criteria and were incorporated into this study. According to a 7:3 ratio, male and female patients in SEER database were randomly divided into the following groups: (I) a male training data set of 10,649 patients; (II) a female training set of 12,101 patients; (III) a male internal testing data set of 4,564 patients; and (IV) a female internal testing data set of 5,187 patients. The included LUAD patients from our hospital were deemed as Chinese external validation cohorts. The gender-specific nomogram models were developed separately based on the male and female LUAD training cohorts, and were verified separately by the male and female LUAD internal and external testing cohorts.

\section{Collection variables}

Information was collected about the following variables for the analysis: overall survival (OS), LCSS, age, gender, tumor site, laterality, tumor differentiation grade, derived AJCC stage, tumor size, historical stage, derived AJCC N stage, surgery information, chemotherapy, and marital status. LCSS was defined as the date of diagnosis to the date of cancer-specific death; deaths by other causes were regarded as the results of other observations. OS was defined as the time from the date of lung cancer diagnosis to death by any cause. The operation information was divided into no surgery, lobectomy (including lobectomy, pneumonectomy, and bronchial sleeve resection), segmentectomy, and wedge resection.

\section{Statistical methods}

Using the Kaplan-Meier method and log-rank tests, we compared all included male and female cancer patients' OS and LCSS rates (see Figure 1). Univariate and multivariate Cox regression models were used to analyze the effects of prognostic factors through R packages "survival", "plyr" (results of univariate cox analyses are provided in Tables $\mathrm{S} 1, \mathrm{~S} 2)$. The variables $(\mathrm{P}<0.05)$ in the multivariable Cox regression analysis among the male and female training cohorts were considered independent prognostic predictors for the establishment of the nomograms. We also calculated the hazard ratios and $95 \%$ confidence intervals using Cox multivariable regression. All the reported $\mathrm{P}$ values were bilateral. $\mathrm{R}$ version 4.0.3 (R) was used to establish and verify the gender-specific nomogram models. Internal verification was evaluated using bootstrap verification. A total of 1,000 re-samplings were performed. The testing cohort was used to check the accuracy of the nomogram models. The calibration curves, concordance index (C-index), decision curve analysis (DCA) curves, receiver operating characteristic (ROC) curves, and area under the curves (AUCs) were used to evaluate the validity of the nomogram models.

The "survival", "forestmodel", "rms", "ggplot2", "cmprsk", and "survivalROC" R packages were used to establish and verify our gender-specific nomogram models.

\section{Results}

\section{Patient characteristics}

This study reviewed 32,654 primary LUAD patients (15,282 males and 17,372 females). Among them, 153 patients in our hospital with postoperative pathological returns of LUAD from January 1, 2015 to October 1, 2016 were enrolled as Chinese validation cohorts. Patients' information is displayed in Table 1. There were no significant differences 

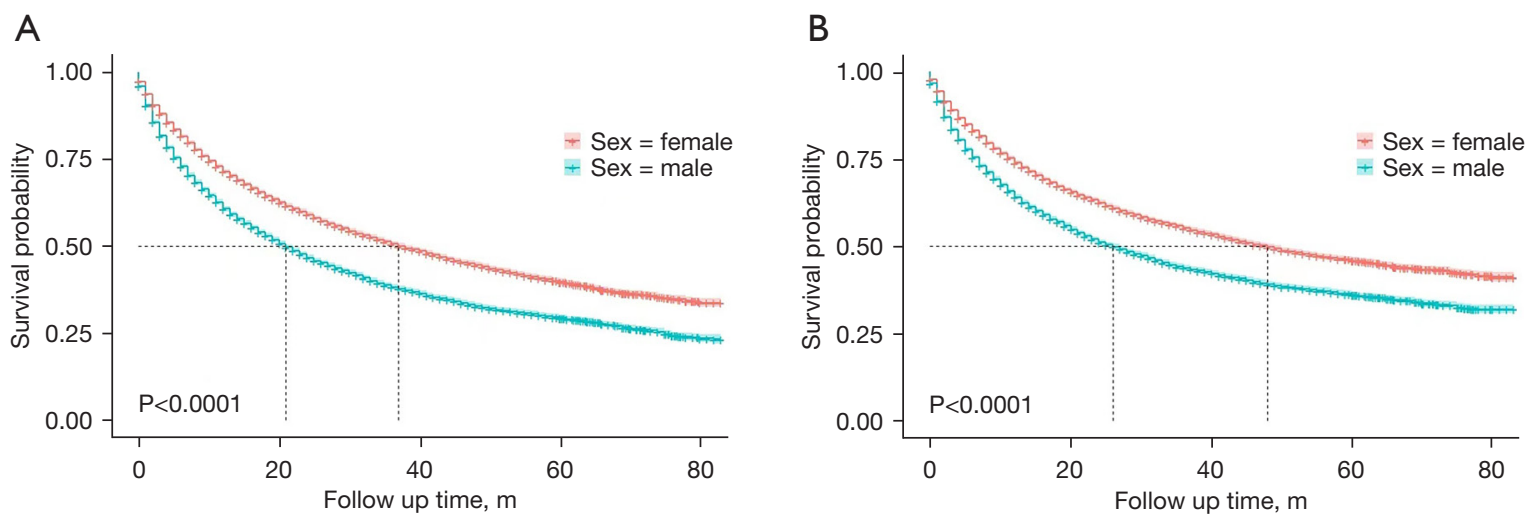

Figure 1 Kaplan-Meier curves for patients with LUAD. (A) OS for male and female LUAD. (B) LCSS for male and female LUAD. LUAD, lung adenocarcinoma; OS, overall survival; LCSS, lung cancer-specific survival.

between the training and validation cohorts.

\section{Nomogram establishment}

LUAD patients from training cohorts $(10,649$ male and 12,101 female LUAD patients) were reckoned to develop the gender-specific nomogram models. The construction of the model is composed of 10 relevant clinical variables which provide personalized prognostic factors for the LCSS of male and female LUAD patients. As Figure 2 shows, 10 variables (age, marital status, tumor site, tumor differentiation grade, derived AJCC stage, tumor size, historic stage, surgery, derived AJCC N stage and chemical treatment) were analyzed as independent factors for LUAD patients. Based on the independent prognostic factors of male and female LUAD, we constructed gender-specific nomogram models for 3- and 5-year LUAD patients' LCSS (see Figure 2A-2D). Results of univariate cox analyses have been provided in Tables $\mathrm{S} 1, \mathrm{~S} 2$.

\section{Nomogram validation}

The gender-specific nomogram models have an excellent performance among LUAD patients, the C-indexes for the female and male LUAD training nomogram models were 0.827 and 0.811 , respectively. Again, C-indexes for the female and male LUAD internal validation models were 0.823 and 0.811 , for external Chinese female and male validation models were 0.89 and 0.831 , respectively. The calibration curves (see Figure 3) showed consistency between predicted and actual patient survival. The ROC curves and AUC values (see Figure 4) were used to evaluate the accuracy of the nomogram models (all AUC values $>8$ ). The DCA curves (see Figure 5) were used to compare our nomogram models with other models. The results showed that our gender-specific models are excellent predictors of LUAD prognosis and can be used to supplement prognostication of TNM stage.

\section{Clinical use}

For example, consider a 60 -year-old married female patient with a regional $3-\mathrm{cm}$ poorly differentiated and AJCC TNM stage II LUAD tumor in the lower lobe, who underwent lobectomy treatment without chemotherapy. The 3- and 5 -year LCSS probabilities were about $64 \%$ and $50 \%$, respectively, and we highly recommended that the patient undergo chemotherapy to increase her LCSS probability. Lobectomy is still considered as the best surgery method for this patient.

\section{Discussion}

Lung cancer is one of the most common cancers and the leading cause of cancer-related death worldwide (1-3). LUAD is the most prevalent subtype of non-small lung cancer (31), and is more common in females than in males (32). In prognosis, the survival rates of male and female LUAD patients differ; in a national cohort study of 23,465 LUAD participants, the prognosis of female LUAD patients in LCSS among TNM stages was kind of higher than that of male patients (19). Meanwhile, female LUAD patients may receive more benefits from using platinumbased chemical drugs (24). Townsend et al. (33) proposed 
Table 1 Characteristics of patients with LUAD

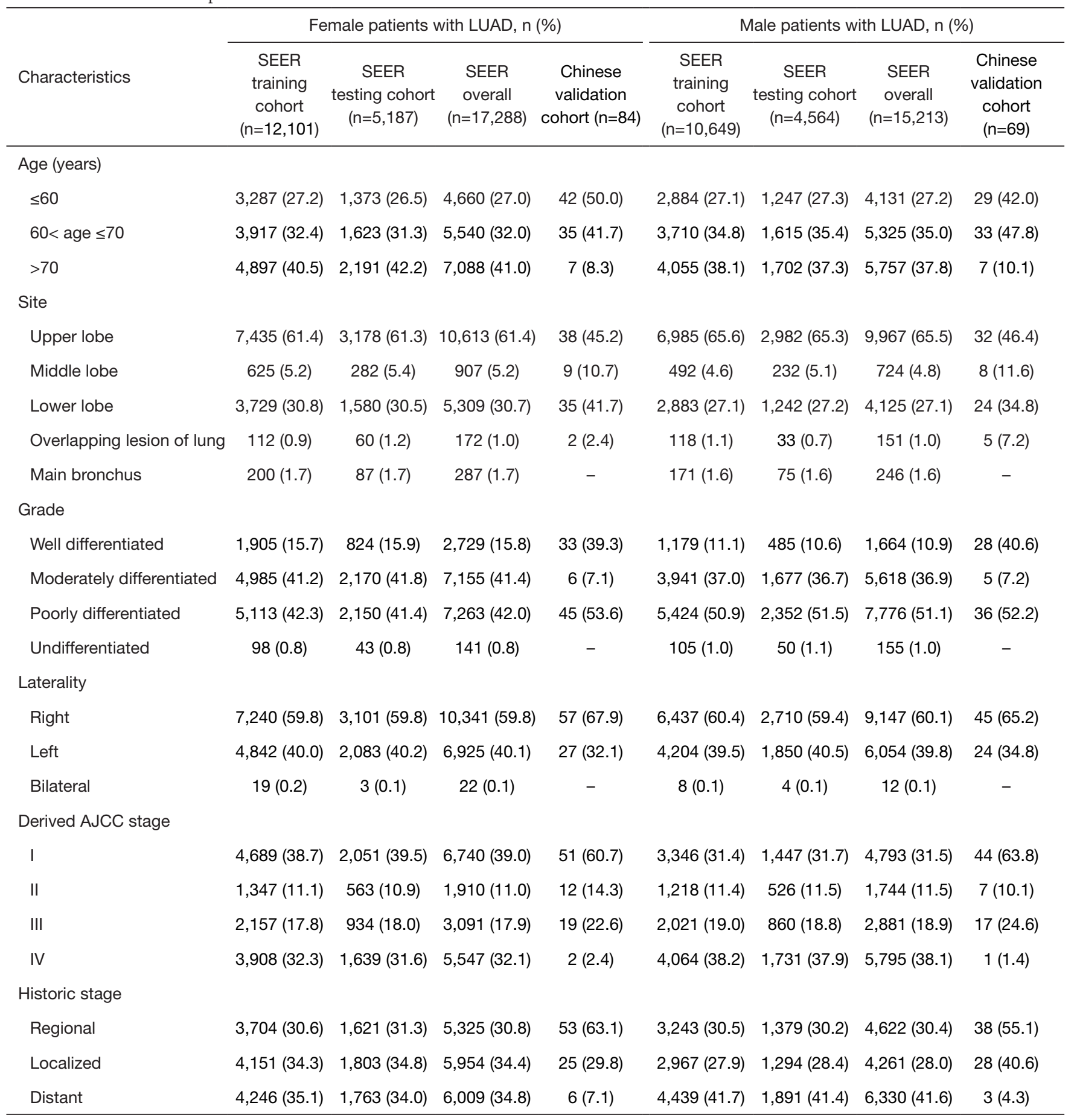

Table 1 (continued) 
Table 1 (continued)

\begin{tabular}{|c|c|c|c|c|c|c|c|c|}
\hline Characteristics & \multicolumn{4}{|c|}{ Female patients with LUAD, n (\%) } & \multicolumn{4}{|c|}{ Male patients with LUAD, n (\%) } \\
\hline \multicolumn{9}{|l|}{ Surgery } \\
\hline Wedge resection & $829(6.9)$ & $394(7.6)$ & $1223(7.1)$ & $1(1.2)$ & $653(6.1)$ & $286(6.3)$ & $939(6.2)$ & - \\
\hline Segmental resection & $219(1.8)$ & $98(1.9)$ & $317(1.8)$ & $3(3.6)$ & $151(1.4)$ & $57(1.2)$ & $208(1.4)$ & $3(4.3)$ \\
\hline Lobectomy & $5,069(41.9)$ & $2,233(43.1)$ & 7,302 (42.2) & $80(95.2)$ & $3,978(37.4)$ & $1,655(36.3)$ & $5,633(37.0)$ & $66(95.7)$ \\
\hline \multicolumn{9}{|l|}{ Derived AJCC N stage } \\
\hline N2 & $3,329(27.5)$ & $1,409(27.2)$ & $4,738(27.4)$ & $8(9.5)$ & $3,182(29.9)$ & $1,367(30.0)$ & 4,549 (29.9) & $6(8.7)$ \\
\hline N3 & $976(8.1)$ & $411(7.9)$ & $1,387(8.0)$ & - & $1,141(10.7)$ & $479(10.5)$ & $1,620(10.6)$ & - \\
\hline \multicolumn{9}{|l|}{ Chemotherapy } \\
\hline No/unknown & $7,117(58.8)$ & $3,049(58.8)$ & $10,166(58.8)$ & $24(28.6)$ & $5,889(55.3)$ & 2,549 (55.9) & $8,438(55.5)$ & $25(36.2)$ \\
\hline Yes & $4,984(41.2)$ & 2,138 (41.2) & 7,122 (41.2) & $60(71.4)$ & $4,760(44.7)$ & 2,015 (44.1) & 6,775 (44.5) & $44(63.8)$ \\
\hline \multicolumn{9}{|l|}{ Size $(\mathrm{cm})$} \\
\hline Size $\leq 3$ & $6,355(52.5)$ & 2,686 (51.8) & $9,041(52.3)$ & 64 (76.2) & 4,666 (43.8) & $1,984(43.5)$ & $6,650(43.7)$ & $55(79.7)$ \\
\hline Size $>3$ & $5,746(47.5)$ & $2,501(48.2)$ & $8,247(47.7)$ & $20(23.8)$ & 5,983 (56.2) & $2,580(56.5)$ & $8,563(56.3)$ & $14(20.3)$ \\
\hline
\end{tabular}

LUAD, lung adenocarcinoma; SEER, Surveillance, Epidemiology, and End Results; AJCC, American Joint Committee on Cancer.

that based on gender molecular differences, sex differences and sex steroids may affect the incidence and outcomes of lung cancer. It should be noted that Behrens et al. consider a higher density of tumor-associated immune infiltrating cells can be found in female LUAD patients' tumor tissue (20). Gender has been considered as an independent prognostic factor for LUAD (18). Sex-related molecular differences can influence the outcome of LUAD (25). Thus, for personalized medicine paradigm, it is reasonable for us to separate LUAD patients by gender to analyze prognosis. Accordingly, excluding gender factors, patients' basic clinical information, AJCC stage, and treatment information are worth considering independent predictors of the possibility of long-term survival and can be used in the establishment of new tools. So the aim of this article is to separately predict the long-term prognosis of LUAD for men and women. For the purpose of achieving accurate individualized treatment, we can incorporate more clinical variables besides age and AJCC TNM stage to make the individualized and more accurate prediction. As previous studies have shown, the support of partners can positively affect patients' expectations and promote their active treatment (34), married LUAD patients have more advantages than unmarried LUAD patients in terms LCSS. Additionally, consistent with the AJCC's TNM system and previous studies $(35,36)$, stage I LUAD patients had a significantly better LCSS rate than stage II/III/IV patients. This study had several limitations. First, it was a retrospective study; thus, selection bias could 
A

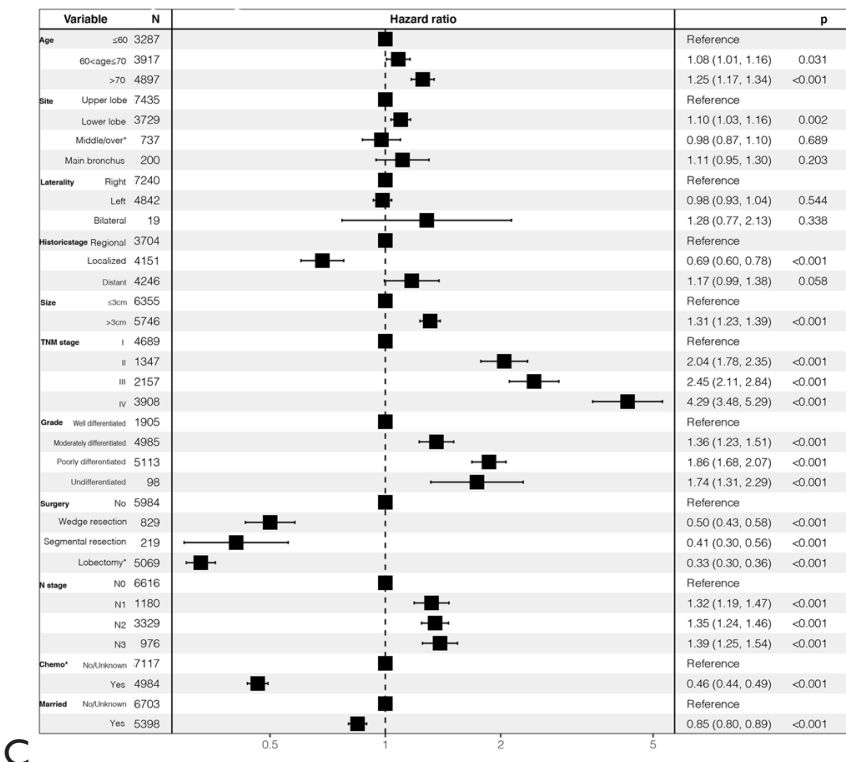

C

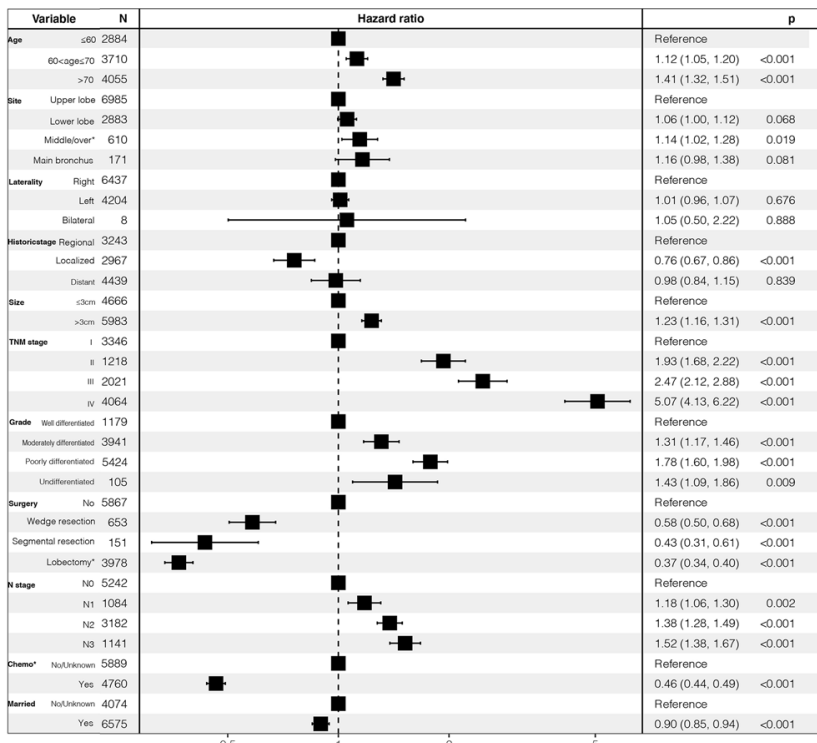

B

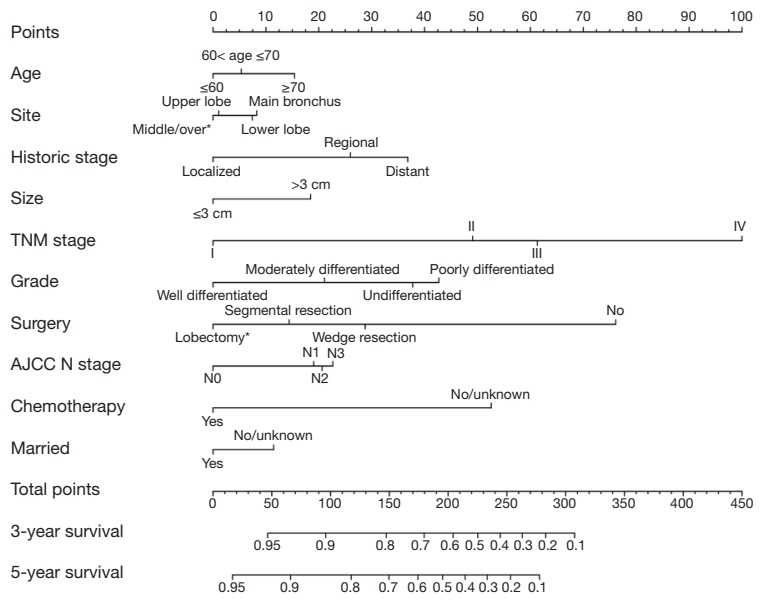

Three-year and five-year Icss prognostic nomogram for female patients with LUAD

D

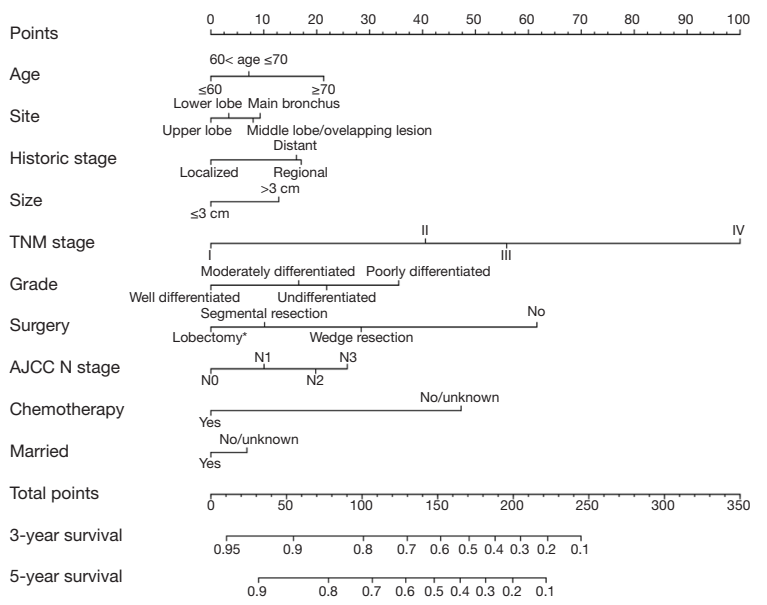

Three-year and five-year lcss prognostic nomogram for male patients with LUAD

Figure 2 Multivariable LCSS Cox regression analysis and nomogram models for female and male LUAD patients. (A,B) Nomogram for female 3- and 5-year LCSS. (C,D) Nomogram for male 3- and 5-year LCSS. Middle/over*, overlapping lesion of lung; lobectomy*, lobectomy + pneumonectomy + bronchial sleeve resection; chemo*, chemotherapy. LCSS, lung cancer-specific survival; LUAD, lung adenocarcinoma; TNM, tumor, node, and metastasis; AJCC, American Joint Committee on Cancer.

not be avoided. Second, many variables affect the prognosis of male and female LUAD patients, including smoking and sex hormone levels, but the SEER database did not provide information on all these variables. Notably, our study also had many advantages. First, this appears to be the first research to separate the prognostic factors of LUAD by gender, and then successfully construct and verify gender- specific nomogram models. Second, this study used largescale cohorts to validate the nomograms. The genderspecific nomogram models provide personalized factors for the prognosis of male and female LUAD patients. The C-indexes of our nomogram models were very high (all $>8$ ), similarly the AUC values of our research were also perfect (all $>8$ ). Thus, our nomogram models are highly reliable. 

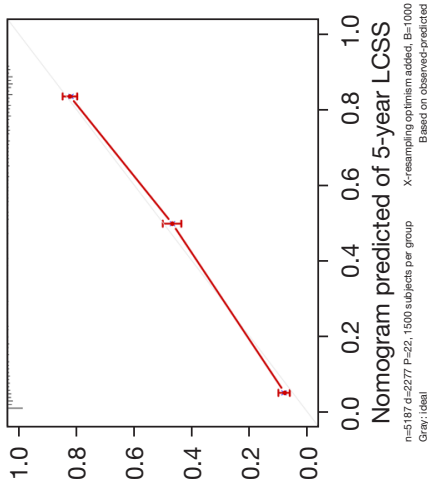

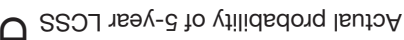
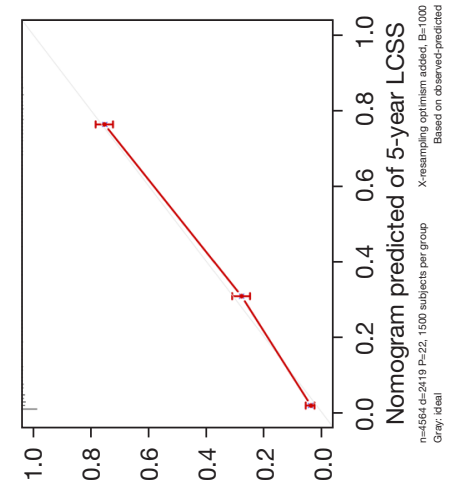

工

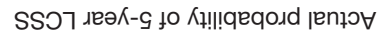
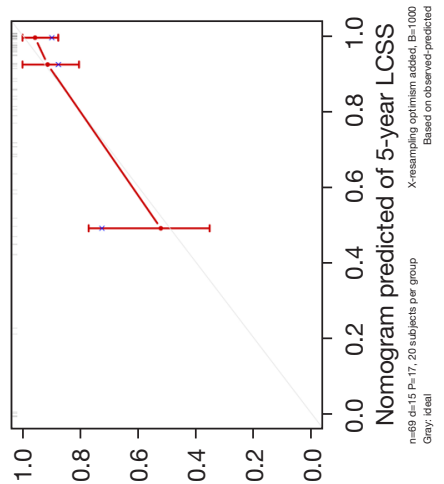

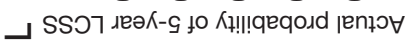

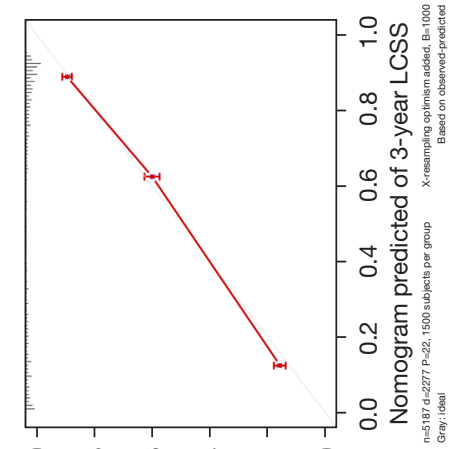

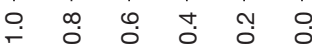

$\cup$ SSวา

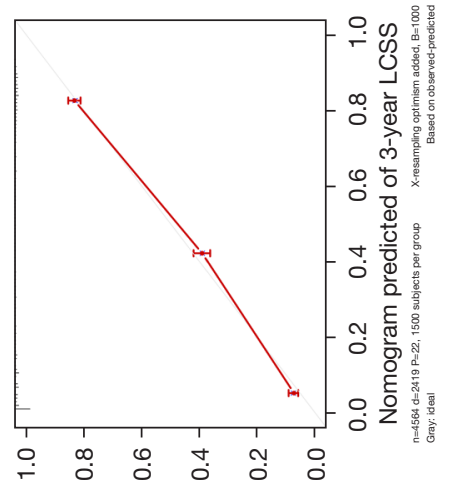

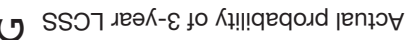
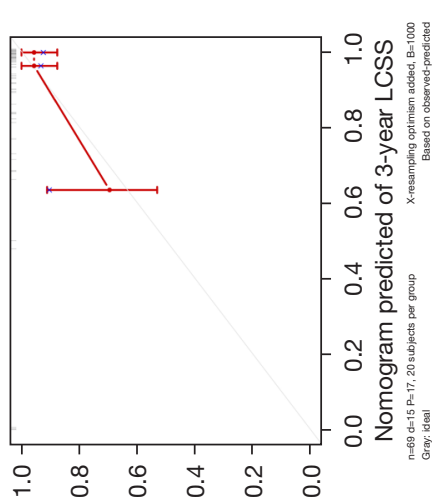

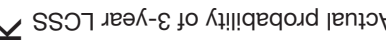

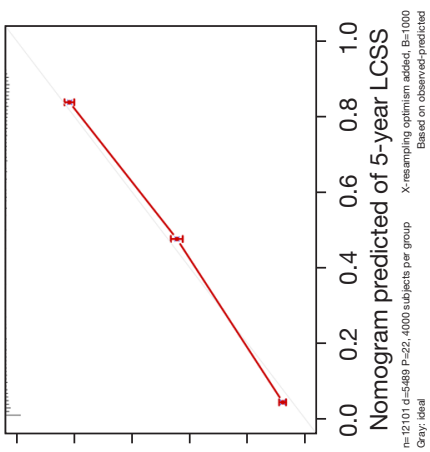

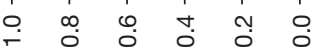

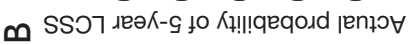
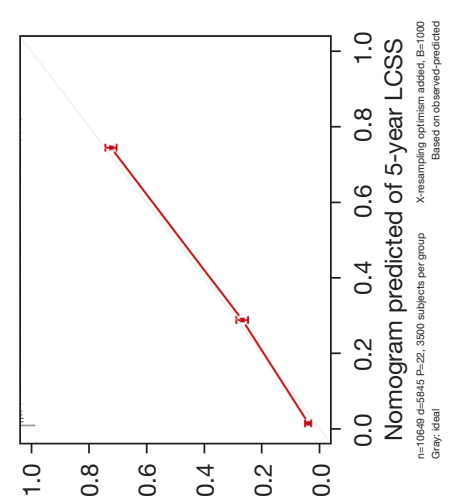

S2วา
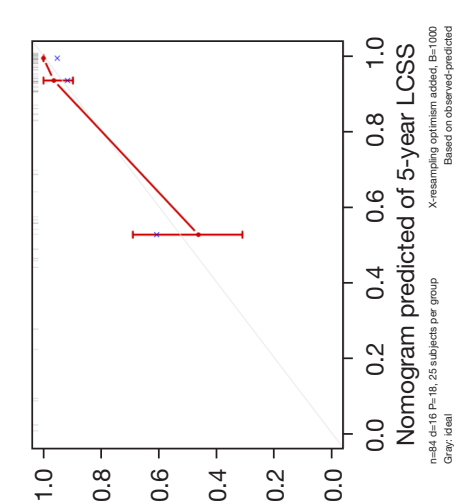

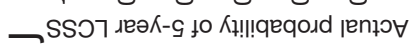

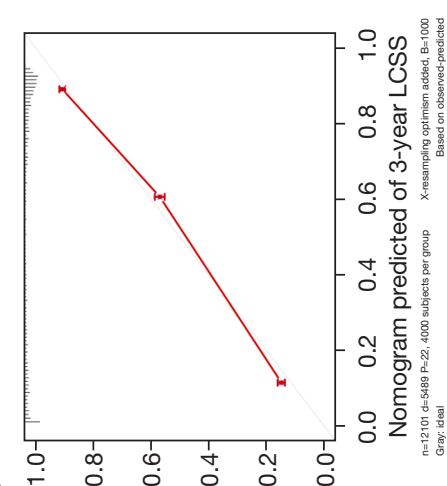

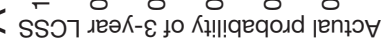

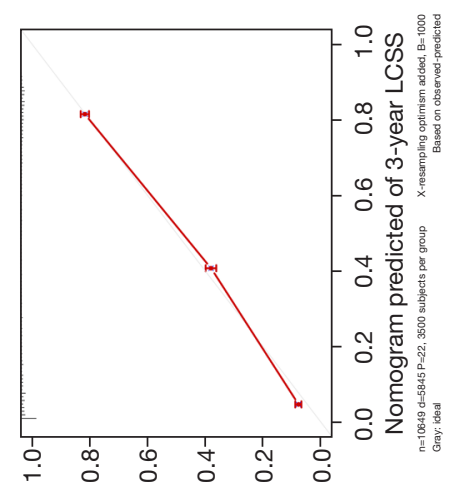

ำ

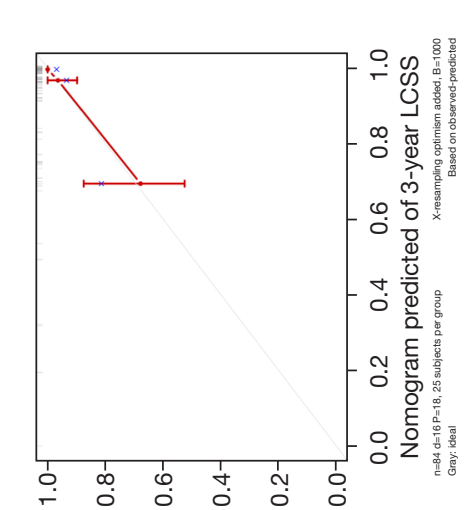

䒕

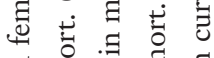
$\exists$ :

记

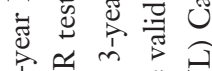
in $\frac{1}{4} \frac{0}{\pi}$

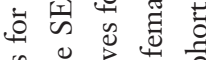

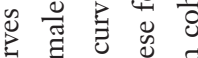

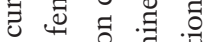

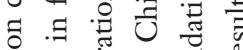
品

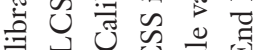

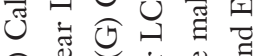
Qิ

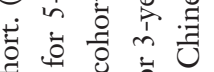

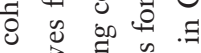

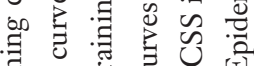

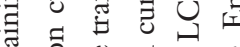
$\because$. ०

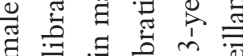
यึ $\exists \widehat{\Theta} \cup$ के

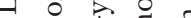

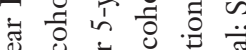

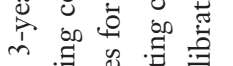

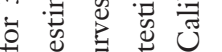
\&

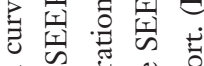
ธี 矛氙

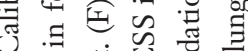
क एं

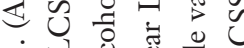
में 0 U్

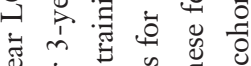

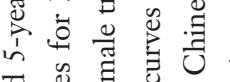

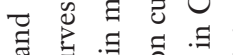

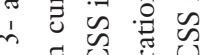
它.

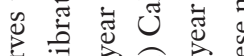

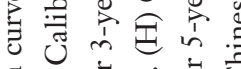

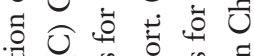

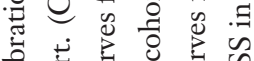
苛 m 000.$]$ ๖

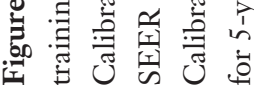



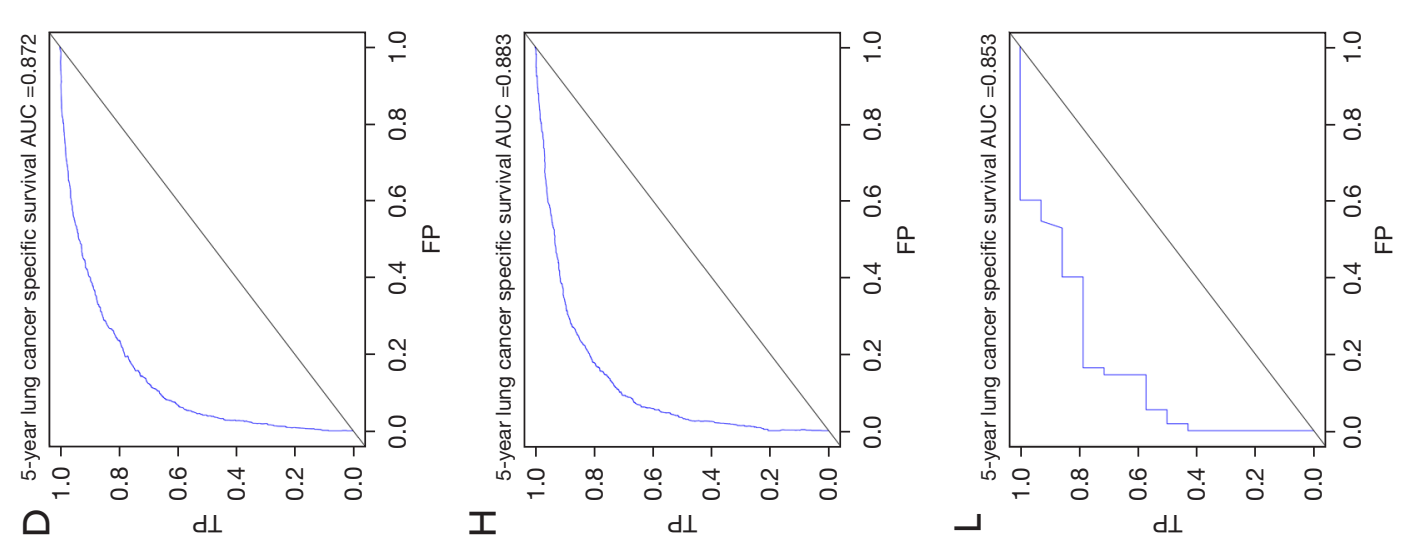

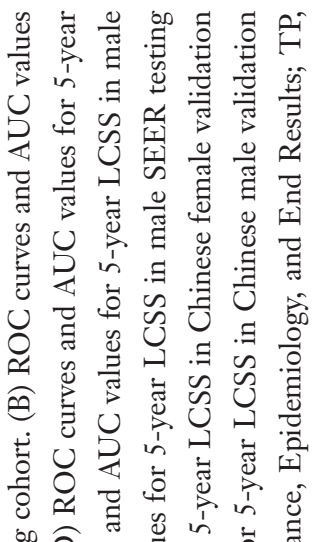
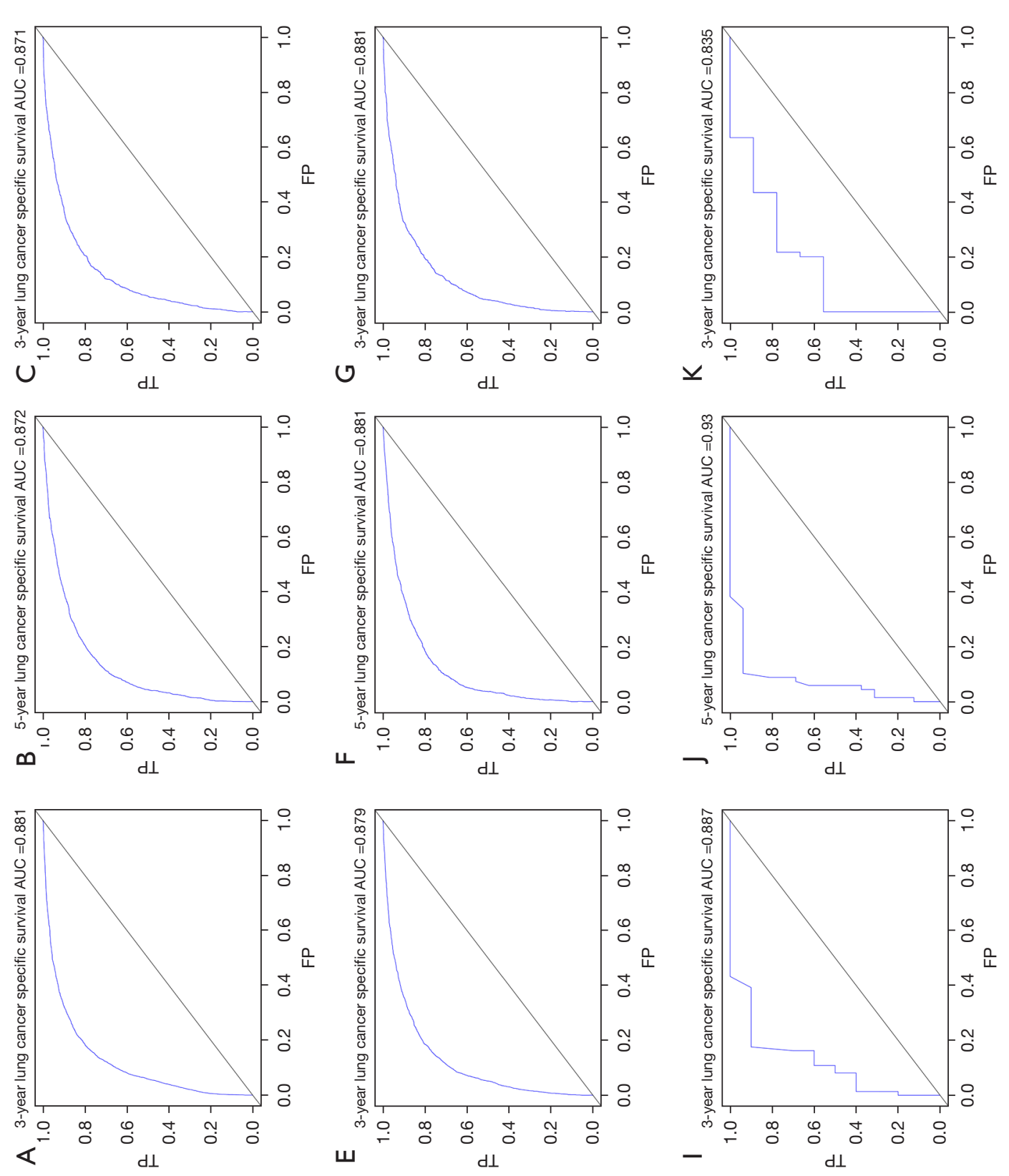

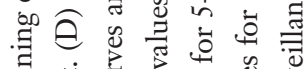

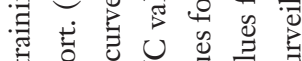

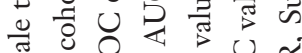
I

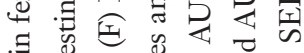

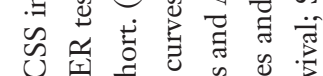

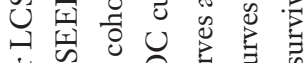
क्षे

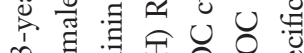

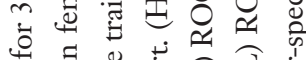

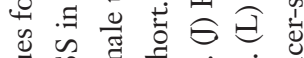

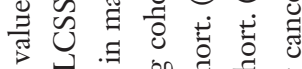

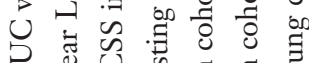
४ 击 के

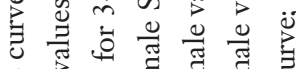
$\cup \circlearrowright ⿻ コ 一 ⿻ 上 丨$

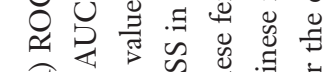

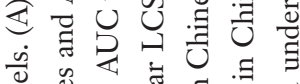

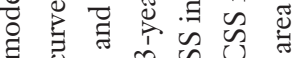

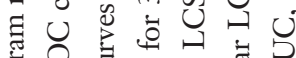

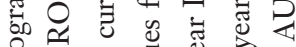

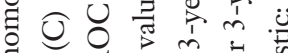

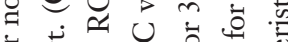

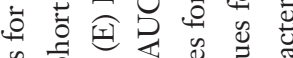

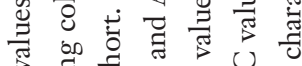
$\rightarrow$ : त्र

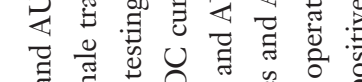

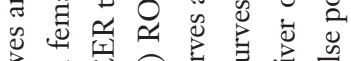

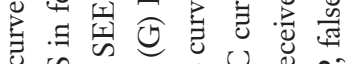
Uू थै

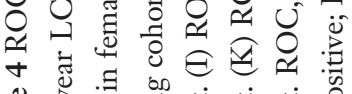

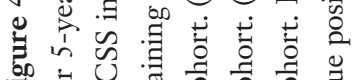
社 
A
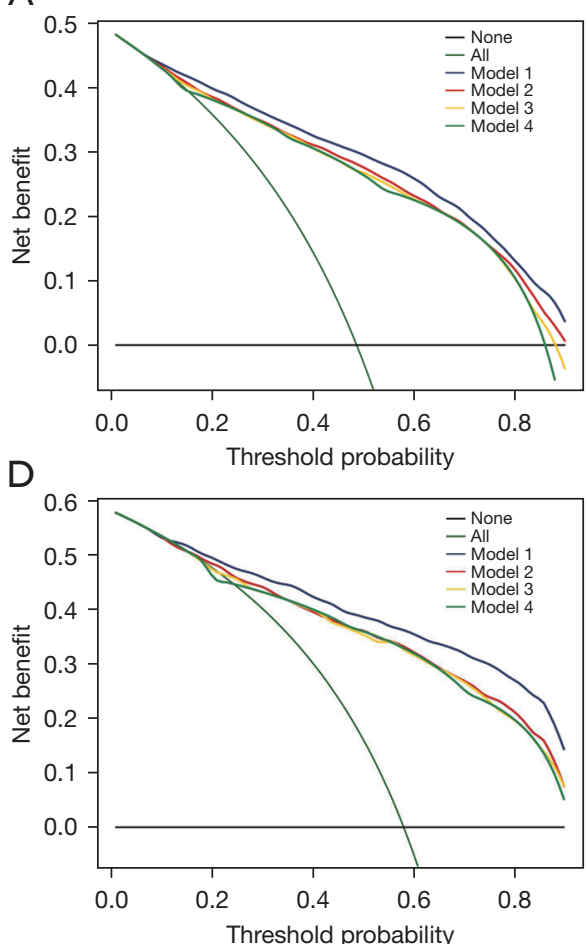

G

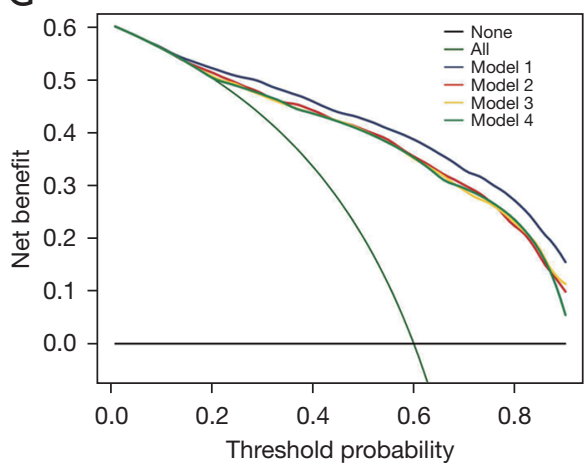

B

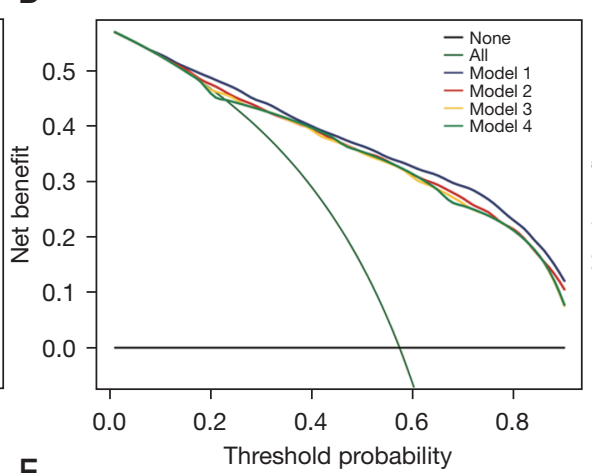

$E$

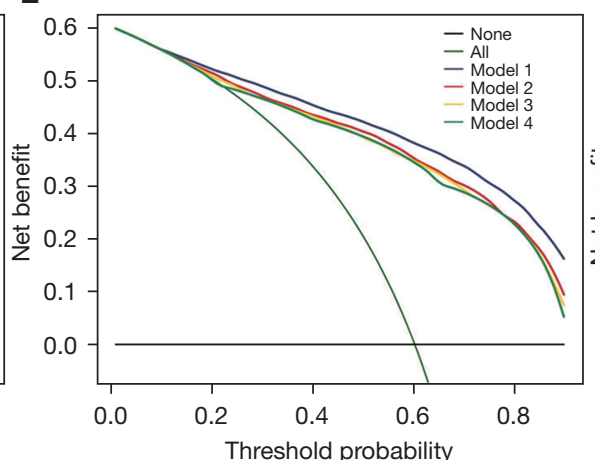

$\mathrm{H}$

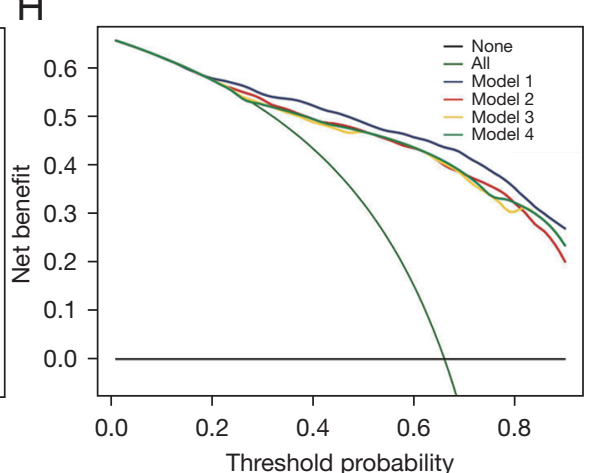

C
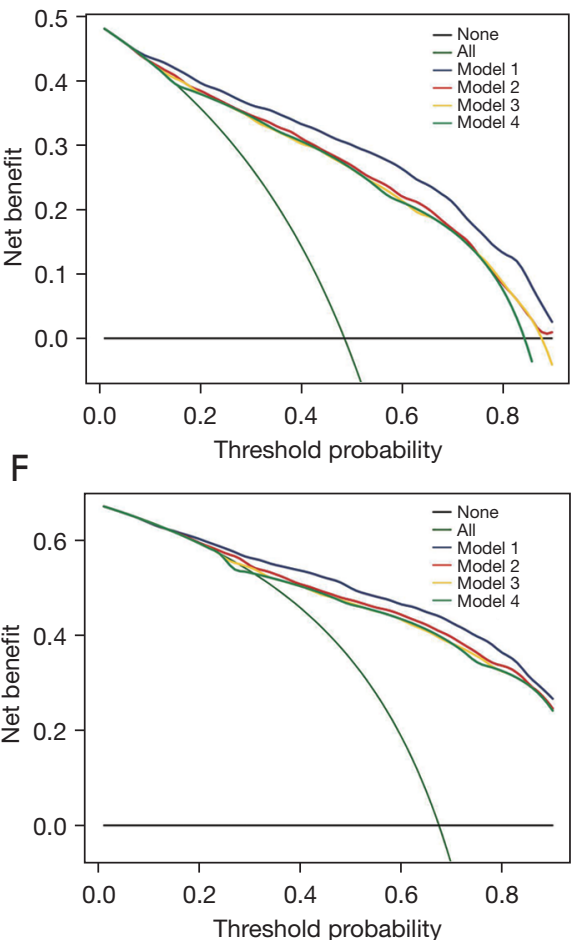

Figure 5 DCA curves for nomogram models and the other three models. (A) DCA curves for 3-year LCSS in female training cohort. (B) DCA curves for 5-year LCSS in female training cohort. (C) DCA curves for 3-year LCSS in female SEER testing cohort. (D) DCA curves for 5-year LCSS in female SEER testing cohort. (E) DCA curves for 3-year LCSS in male training cohort. (F) DCA curves for 5-year LCSS in male training cohort. (G) DCA curves for 3-year LCSS in male SEER testing cohort. (H) DCA curves for 5-year LCSS in male SEER testing cohort. Mode 1: gender-specific nomogram model; model 2: AJCC TNM stage + historic stage + tumor differentiation grade + age + tumor size; model 3: tumor differentiation grade + AJCC TNM stage; model 4: AJCC TNM stage. DCA, decision curve analysis; LCSS, lung cancer-specific survival; SEER, Surveillance, Epidemiology, and End Results; AJCC, American Joint Committee on Cancer; TNM, tumor, node, and metastasis.

\section{Conclusions}

We developed gender-specific nomogram models that can be used as a supplement to the AJCC TNM stages to accurately predict the prognosis of male and female LUAD patients. Excluding gender factors, these nomogram models may help clinicians to accurately predict the prognosis of LUAD patients and make appropriate decisions about treatment strategies. 


\section{Acknowledgments}

The authors would like to thank the SEER program for open access to the database.

Funding: This study was supported by the Project of Tianjin Key Clinical Disciplines and the Project of Tianjin Health Commission (grant No. ZD20023) and the Project of Tianjin Science and Technology Innovation Bureau (grant No. 20JCYBJC01350).

\section{Footnote}

Reporting Checklist: The authors have completed the TRIPOD reporting checklist. Available at https://dx.doi. org/10.21037/atm-21-5367

Data Sharing Statement: Available at https://dx.doi. org/10.21037/atm-21-5367

Conflicts of Interest: All authors have completed the ICMJE uniform disclosure form (available at https://dx.doi. org/10.21037/atm-21-5367). All authors report that this study was supported by the Project of Tianjin Key Clinical Disciplines and the Project of Tianjin Health Commission (grant No. ZD20023) and the Project of Tianjin Science and Technology Innovation Bureau (grant No. 20JCYBJC01350). The authors have no other conflicts of interest to declare.

Ethical Statement: The authors are accountable for all aspects of the work in ensuring that questions related to the accuracy or integrity of any part of the work are appropriately investigated and resolved. All of the procedures performed in this study were conducted in accordance with the Declaration of Helsinki (as revised in 2013), and written informed consents have been provided by all included patients from Tianjin Chest Hospital. The study was approved by institutional ethics board of Tianjin chest Hospital (ethical approval number: 2021LW-016).

Open Access Statement: This is an Open Access article distributed in accordance with the Creative Commons Attribution-NonCommercial-NoDerivs 4.0 International License (CC BY-NC-ND 4.0), which permits the noncommercial replication and distribution of the article with the strict proviso that no changes or edits are made and the original work is properly cited (including links to both the formal publication through the relevant DOI and the license).
See: https://creativecommons.org/licenses/by-nc-nd/4.0/.

\section{References}

1. Sung H, Ferlay J, Siegel RL, et al. Global Cancer Statistics 2020: GLOBOCAN Estimates of Incidence and Mortality Worldwide for 36 Cancers in 185 Countries. CA Cancer J Clin 2021;71:209-49.

2. Ferlay J, Colombet M, Soerjomataram I, et al. Cancer statistics for the year 2020: an overview. Int J Cancer 2021. [Epub ahead of print]. doi: 10.1002/ijc.33588.

3. Siegel RL, Miller KD, Fuchs HE, et al. Cancer Statistics, 2021. CA Cancer J Clin 2021;71:7-33.

4. Duma N. Lung-cancer researchers and clinicians must pay more attention to women. Nature 2020;587:S13.

5. Cho J, Choi SM, Lee J, et al. Proportion and clinical features of never-smokers with non-small cell lung cancer. Chin J Cancer 2017;36:20.

6. Yano T, Miura N, Takenaka T, et al. Never-smoking nonsmall cell lung cancer as a separate entity: clinicopathologic features and survival. Cancer 2008;113:1012-8.

7. Jin K, Hung RJ, Thomas S, et al. Hormonal factors in association with lung cancer among Asian women: A pooled analysis from the International Lung Cancer Consortium. Int J Cancer 2021;148:2241-54.

8. Liau CS, Mogan P, Thomas W. Oestrogen actions contribute to female gender-specific risks in the development of lung carcinoma. J Steroid Biochem Mol Biol 2021;208:105786.

9. Smida T, Bruno TC, Stabile LP. Influence of Estrogen on the NSCLC Microenvironment: A Comprehensive Picture and Clinical Implications. Front Oncol 2020;10:137.

10. Saito S, Espinoza-Mercado F, Liu H, et al. Current status of research and treatment for non-small cell lung cancer in never-smoking females. Cancer Biol Ther 2017;18:359-68.

11. Huang Q, Zhang Z, Liao Y, et al. 17 $\beta$-estradiol upregulates IL6 expression through the ER $\beta$ pathway to promote lung adenocarcinoma progression. J Exp Clin Cancer Res 2018;37:133.

12. He J, Li W, Li Y, et al. Construction of a prognostic model for lung adenocarcinoma based on bioinformatics analysis of metabolic genes. Transl Cancer Res 2020;9:3518-38.

13. Wang X, Shi B, Zhao Y, et al. HKDC1 promotes the tumorigenesis and glycolysis in lung adenocarcinoma via regulating AMPK/mTOR signaling pathway. Cancer Cell Int 2020;20:450.

14. Wang X, Yao S, Xiao Z, et al. Development and validation 
of a survival model for lung adenocarcinoma based on autophagy-associated genes. J Transl Med 2020;18:149.

15. Wakelee HA, Chang ET, Gomez SL, et al. Lung cancer incidence in never smokers. J Clin Oncol 2007;25:472-8.

16. Pallis AG, Syrigos KN. Lung cancer in never smokers: disease characteristics and risk factors. Crit Rev Oncol Hematol 2013;88:494-503.

17. Yano T, Haro A, Shikada Y, et al. Non-small cell lung cancer in never smokers as a representative 'non-smokingassociated lung cancer': epidemiology and clinical features. Int J Clin Oncol 2011;16:287-93.

18. Pitz MW, Musto G, Navaratnam S. Sex as an independent prognostic factor in a population-based non-small cell lung cancer cohort. Can Respir J 2013;20:30-4.

19. Radkiewicz C, Dickman PW, Johansson ALV, et al. Sex and survival in non-small cell lung cancer: A nationwide cohort study. PLoS One 2019;14:e0219206.

20. Behrens C, Rocha P, Parra ER, et al. Female Gender Predicts Augmented Immune Infiltration in Lung Adenocarcinoma. Clin Lung Cancer 2021;22:e415-24.

21. Woolston A, Sintupisut N, Lu TP, et al. Putative effectors for prognosis in lung adenocarcinoma are ethnic and gender specific. Oncotarget 2015;6:19483-99.

22. Chen W, Xin B, Pang H, et al. Downregulation of estrogen receptor $\beta$ inhibits lung adenocarcinoma cell growth. Oncol Rep 2019;41:2967-74.

23. Skov BG, Fischer BM, Pappot H. Oestrogen receptor beta over expression in males with non-small cell lung cancer is associated with better survival. Lung Cancer 2008;59:88-94.

24. Wheatley-Price P, Blackhall F, Lee SM, et al. The influence of sex and histology on outcomes in non-smallcell lung cancer: a pooled analysis of five randomized trials. Ann Oncol 2010;21:2023-8.

25. Pérez-Díez I, Hidalgo MR, Malmierca-Merlo P, et al. Functional Signatures in Non-Small-Cell Lung Cancer: A Systematic Review and Meta-Analysis of Sex-Based Differences in Transcriptomic Studies. Cancers (Basel) 2021;13:143.

26. Zhang R, Xu M, Liu X, et al. Establishment and validation of a nomogram model for predicting the survival

Cite this article as: Wen H, Lin X, Sun D. Gender-specific nomogram models to predict the prognosis of male and female lung adenocarcinoma patients: a population-based analysis. Ann Transl Med 2021;9(22):1654. doi: 10.21037/atm-21-5367 probability of differentiated thyroid carcinoma patients: a comparison with the eighth edition AJCC cancer staging system. Endocrine 2021;74:108-19.

27. Liu J, Nie S, Li S, et al. Methylation-driven genes and their prognostic value in cervical squamous cell carcinoma. Ann Transl Med 2020;8:868.

28. Lv Z, Liang Y, Liu H, et al. Association of chemotherapy with survival in stage II colon cancer patients who received radical surgery: a retrospective cohort study. BMC Cancer 2021;21:306.

29. Wang SB, Qi WX, Chen JY, et al. Competing risk nomogram predicting initial loco-regional recurrence in gastric cancer patients after D2 gastrectomy. Radiat Oncol 2019;14:128.

30. Balachandran VP, Gonen M, Smith JJ, et al. Nomograms in oncology: more than meets the eye. Lancet Oncol 2015;16:e173-80.

31. Behera M, Owonikoko TK, Gal AA, et al. Lung Adenocarcinoma Staging Using the 2011 IASLC/ATS/ ERS Classification: A Pooled Analysis of Adenocarcinoma In Situ and Minimally Invasive Adenocarcinoma. Clin Lung Cancer 2016;17:e57-64.

32. Sui Q, Liang J, Hu Z, et al. Genetic and microenvironmental differences in non-smoking lung adenocarcinoma patients compared with smoking patients. Transl Lung Cancer Res 2020;9:1407-21.

33. Townsend EA, Miller VM, Prakash YS. Sex differences and sex steroids in lung health and disease. Endocr Rev 2012;33:1-47.

34. Kanters AE, Morris AM, Abrahamse PH, et al. The Effect of Peer Support on Colorectal Cancer Patients' Adherence to Guideline-Concordant Multidisciplinary Care. Dis Colon Rectum 2018;61:817-23.

35. Woodard GA, Jones KD, Jablons DM. Lung Cancer Staging and Prognosis. Cancer Treat Res 2016;170:47-75.

36. Cagle PT. Lung Carcinoma Staging Problems. Surg Pathol Clin 2010;3:61-9.

(English Language Editor: L. Huleatt) 
Table S1 Univariate cox analyses for male LUAD patients

\begin{tabular}{|c|c|c|c|}
\hline Characteristics & $\mathrm{HR}$ & $\mathrm{P}$ & $\mathrm{Cl}$ \\
\hline \multicolumn{4}{|l|}{ Site } \\
\hline \multicolumn{4}{|l|}{ Upper lobe } \\
\hline Lower lobe & 1.07 & 0.019 & $1.01-1.14$ \\
\hline Middle lobe/overlapping & 1.09 & 0.124 & $0.98-1.22$ \\
\hline Main bronchus & 2.38 & 0 & $2.01-2.82$ \\
\hline \multicolumn{4}{|l|}{ Grade } \\
\hline \multicolumn{4}{|l|}{ Well grade } \\
\hline Moderate grade & 1.58 & 0 & $1.42-1.77$ \\
\hline Poor grade & 2.99 & 0 & $2.69-3.32$ \\
\hline Undifferentiated grade & 2.65 & 0 & $2.04-3.45$ \\
\hline \multicolumn{4}{|l|}{ Laterality } \\
\hline \multicolumn{4}{|l|}{ Right } \\
\hline Left & 1.03 & 0.351 & $0.97-1.08$ \\
\hline \multicolumn{4}{|l|}{ Derived AJCC stage } \\
\hline \multicolumn{4}{|l|}{ I } \\
\hline II & 2.01 & 0 & $1.79-2.26$ \\
\hline III & 4.24 & 0 & $3.86-4.65$ \\
\hline IV & 10.67 & 0 & $9.83-11.6$ \\
\hline \multicolumn{4}{|l|}{ Derived AJCC N stage } \\
\hline \multicolumn{4}{|l|}{ No } \\
\hline N1 & 1.83 & 0 & $1.67-2$ \\
\hline N2 & 3.65 & 0 & $3.44-3.88$ \\
\hline N3 & 4.82 & 0 & $4.46-5.22$ \\
\hline \multicolumn{4}{|l|}{ Surgery method } \\
\hline \multicolumn{4}{|l|}{ No surgery } \\
\hline Wedge resection & 0.23 & 0 & $0.2-0.26$ \\
\hline Segmental resection & 0.15 & 0 & $0.11-0.21$ \\
\hline Lobectomy & 0.15 & 0 & $0.14-0.17$ \\
\hline \multicolumn{4}{|l|}{ Chemotherapy } \\
\hline \multicolumn{4}{|l|}{ No/unknown } \\
\hline Yes & 1.51 & 0 & $1.43-1.59$ \\
\hline \multicolumn{4}{|l|}{ Tumor size } \\
\hline \multicolumn{4}{|l|}{$\leq 3 \mathrm{~cm}$} \\
\hline$>3 \mathrm{~cm}$ & 2.54 & 0 & $2.4-2.69$ \\
\hline \multicolumn{4}{|l|}{ Age } \\
\hline \multicolumn{4}{|l|}{$\leq 60$} \\
\hline $60<$ age $\leq 70$ & 0.91 & 0.006 & $0.85-0.97$ \\
\hline$>70$ & 1.17 & 0 & $1.1-1.25$ \\
\hline \multicolumn{4}{|l|}{ Marital } \\
\hline \multicolumn{4}{|l|}{ No married/unknown } \\
\hline Married & 0.83 & 0 & $0.79-0.88$ \\
\hline \multicolumn{4}{|l|}{ Historic stage } \\
\hline \multicolumn{4}{|l|}{ Regional } \\
\hline Local & 0.42 & 0 & $0.38-0.46$ \\
\hline Distant & 3.77 & 0 & $3.54-4.01$ \\
\hline
\end{tabular}

LUAD, lung adenocarcinoma; AJCC, American Joint Committee on Cancer. 


\begin{tabular}{|c|c|c|c|}
\hline Characteristics & $\mathrm{HR}$ & $P$ & $\mathrm{Cl}$ \\
\hline \multicolumn{4}{|l|}{ Site } \\
\hline \multicolumn{4}{|l|}{ Upper lobe } \\
\hline Lower lobe & 1.04 & 0.238 & $0.98-1.1$ \\
\hline Middle lobe/overlapping & 1.02 & 0.78 & $0.91-1.14$ \\
\hline Main bronchus & 2.79 & 0 & $2.38-3.27$ \\
\hline \multicolumn{4}{|l|}{ Grade } \\
\hline \multicolumn{4}{|l|}{ Well grade } \\
\hline Moderate grade & 1.73 & 0 & $1.56-1.91$ \\
\hline Poor grade & 3.52 & 0 & $3.19-3.89$ \\
\hline Undifferentiated grade & 3.22 & 0 & $2.44-4.24$ \\
\hline \multicolumn{4}{|l|}{ Laterality } \\
\hline \multicolumn{4}{|l|}{ Right } \\
\hline Left & 1.02 & 0.499 & $0.97-1.07$ \\
\hline \multicolumn{4}{|l|}{ Derived AJCC stage } \\
\hline \multicolumn{4}{|l|}{ I } \\
\hline ॥ & 2.6 & 0 & $2.32-2.92$ \\
\hline III & 4.86 & 0 & $4.43-5.34$ \\
\hline IV & 12.5 & 0 & $11.51-13.58$ \\
\hline \multicolumn{4}{|l|}{ Derived AJCC N stage } \\
\hline \multicolumn{4}{|l|}{ No } \\
\hline $\mathrm{N} 1$ & 2.33 & 0 & $2.13-2.56$ \\
\hline N2 & 4.17 & 0 & $3.92-4.44$ \\
\hline N3 & 5.72 & 0 & $5.26-6.23$ \\
\hline \multicolumn{4}{|l|}{ Surgery method } \\
\hline \multicolumn{4}{|l|}{ No surgery } \\
\hline Wedge resection & 0.19 & 0 & $0.17-0.22$ \\
\hline Segmental resection & 0.14 & 0 & $0.11-0.19$ \\
\hline Lobectomy & 0.14 & 0 & $0.13-0.15$ \\
\hline \multicolumn{4}{|l|}{ Chemotherapy } \\
\hline \multicolumn{4}{|l|}{ No/unknown } \\
\hline Yes & 1.86 & 0 & $1.76-1.96$ \\
\hline \multicolumn{4}{|l|}{ Tumor size } \\
\hline \multicolumn{4}{|l|}{$\leq 3 \mathrm{~cm}$} \\
\hline$>3 \mathrm{~cm}$ & 2.9 & 0 & $2.74-3.07$ \\
\hline \multicolumn{4}{|l|}{ Age } \\
\hline \multicolumn{4}{|l|}{$\leq 60$} \\
\hline $60<$ age $\leq 70$ & 0.95 & 0.194 & $0.89-1.02$ \\
\hline$>70$ & 1.19 & 0 & $1.12-1.27$ \\
\hline \multicolumn{4}{|l|}{ Marital } \\
\hline \multicolumn{4}{|l|}{ No married/unknown } \\
\hline Married & 0.84 & 0 & $0.8-0.89$ \\
\hline \multicolumn{4}{|l|}{ Historic stage } \\
\hline \multicolumn{4}{|l|}{ Regional } \\
\hline Local & 0.35 & 0 & $0.32-0.39$ \\
\hline Distant & 3.91 & 0 & $3.68-4.17$ \\
\hline
\end{tabular}

LUAD, lung adenocarcinoma; AJCC, American Joint Committee on Cancer. 